\title{
Evaluation of different API systems for identification of porcine Pasteurella multocida isolates
}

\author{
Y.A. Vera Lizarazo, E.F. Rodríguez Ferri, C.B. Gutiérrez Martín* \\ Department of Animal Health, Section of Microbiology and Immunology, Faculty of Veterinary Medicine, University of León, 24007 León, Spain
}

Accepted 5 February 2008

\begin{abstract}
An exhaustive biochemical characterisation of 60 porcine Pasteurella multocida clinical isolates recovered from lesions indicative of pneumonia, previously confirmed by PCR and all belonging to the capsular serogroup A, was performed by means of four commercial systems. The API 20 NE correctly identified almost all isolates $(95 \%)$, but only $60 \%$ could be ascribed to this species by the API $20 \mathrm{E}$ method. The high diversity exhibited by the API 50CHB/E system, with six different patterns, does not advise its use as additional system for a definitive identification at the species level, but this method could be a potential tool for characterising $P$. multocida isolates below this level. The more uniform reactions yielded by the API ZYM test make this system helpful in the confirmatory identification of this organism. The high variability (20 profiles) obtained when the four systems are taken together also suggests their usefulness for epidemiological purposes in order to sub-type $P$. multocida isolates.
\end{abstract}

(C) 2008 Elsevier Ltd. All rights reserved.

Keywords: Pasteurella multocida; Swine; Biochemical characterisation; Identification

Although Pasteurella multocida is part of the commensal flora of the upper respiratory tract of swine, it may induce pneumonia in growing and finishing pigs to the extent that pneumonic pasteurellosis is one of the most common diseases of intensively housed pigs. The disease is often associated with other important bacterial respiratory infections (enzootic pneumonia caused by Mycoplasma hyopneumoniae and pleuropneumonia caused by Actinobacillus pleuropneumoniae), and results in a disease condition described as chronic respiratory disease. In this disorder, P. multocida usually acts as a secondary pathogen, invading lungs injured by other organisms (Pijoan, 1992). In contrast, systemic pasteurellosis is not a frequently diagnosed disease in pigs, although outbreaks of acute septicaemia have been reported (Blackall et al., 2000). In addition, P. multocida may induce progressive atrophic rhinitis in association with Bordetella bronchiseptica (De Jong, 1992).

\footnotetext{
* Corresponding author. Tel.: +34 987291 203; fax: +34 987291304.

E-mail address: cbgutm@unileon.es (C.B. Gutiérrez Martín).
}

In this study, we report an exhaustive biochemical characterisation of $P$. multocida clinical isolates recovered from porcine lungs, and evaluate the accuracy of API 20E in their identification, as well as the capacity of API 50CHB/E and API ZYM as additional systems for a confirmatory identification. The API 20NE, used as routine system in clinical laboratories, was also included.

A total of 60 P. multocida isolates were recovered from swine with lesions indicative of pneumonia coming from 23 farms in northeastern, northwestern and central Spain between 2003 and 2004. The bacteria were first isolated on Columbia agar with 5\% sheep blood (bioMérieux, Lyon, France), and the mucous non-haemolitic colonies were further plated on a selective medium for P. multocida (Avril et al., 1990): Müeller-Hinton agar (Pronadisa, Spain) with 5\% defibrinated horse blood (bioMérieux), amikacin $(2 \mathrm{mg} / \mathrm{l})$ (Sigma Chemical Co., St. Louis, Mo), vancomycin (4 mg/l) (Sigma), and amphotericin B (4 mg/ 1) (Sigma). The isolates were genetically confirmed by use of a multiplex PCR assay (Townsend et al., 2001), and all of them were found to belong to capsular serogroup A. 
Each clinical isolate was processed using the API 20E, API 20NE, API 50CHB/E and API ZYM systems (bioMérieux), along with the P. multocida $\mathrm{NCTC} 10322^{\mathrm{T}}$ reference strain. In addition, control strains were used as recommended by the manufacturer (Klebsiella pneumoniae subsp. pneumoniae ATCC 35657 for API 20E and API 50CHB/E, Aeromonas hydrophila ATCC 35654 for API 20NE, and Bacteroides thetaiotaomicron ATCC 8492 for API ZYM). Each P. multocida isolate was inoculated into $0.85 \% \mathrm{NaCl}$, and turbidity was adjusted to $0.5 \mathrm{MacF}$ arland standard for API 20E and API 20NE; to 2 for API 50CHB/ $\mathrm{E}$, and to 5 for API ZYM. The inoculum was distributed into test strips according to the manufacturer's instructions. API ZYM strips were read after a 4-h incubation at $37^{\circ} \mathrm{C}$; API $20 \mathrm{E}$ strips after a 24 -h incubation at $37^{\circ} \mathrm{C}$; and API $20 \mathrm{NE}$ and API $50 \mathrm{CHB} / \mathrm{E}$ strips after a 48 -h incubation at $30^{\circ} \mathrm{C}$ and $37^{\circ} \mathrm{C}$, respectively. Biochemical reactions were read as positive or negative, translated into numerical profiles (for API 20E and API20NE), and interpreted with the software APILAB Plus update 3.2.2. For API ZYM, the reactions were considered only positive for a strong activity ( $\geqslant 30 \mathrm{~nm}$ of hydrolysed substrate), according to the recommendations of the manufacturer. The four commercial systems were tested for their reproducibility, by use of $P$. multocida NCTC $10322^{\mathrm{T}}$, which was replicated five times, as previously described (Purdy et al., 1997).

The API 20E profiles allowed us to establish three phenotypic patterns for the $P$. multocida isolates (Table 1). The profile with the highest number of them was pattern I $(60 \%)$, followed by patterns II $(26.7 \%)$ and III $(13.3 \%)$. Pattern I also included the reference strain NCTC $10322^{\mathrm{T}}$. The API 20E strips correctly identified at the species level only the isolates belonging to pattern I (0040524;

Table 1

Biochemical patterns of 60 porcine clinical isolates of Pasteurella multocida yielded by the API 20E system

\begin{tabular}{llll}
\hline & Pattern & & \\
\cline { 2 - 4 } & $\mathrm{I}$ & $\mathrm{II}$ & $\mathrm{III}$ \\
\hline Ornithine decarboxylase & - & + & - \\
Indole production & + & - & + \\
$\begin{array}{l}\text { Fermentation of } \\
\text { mannitol, sorbitol and }\end{array}$ & + & - & - \\
$\quad$ sucrose & & & \\
Reduction of nitrates & + & - & + \\
No. (\%) of isolates & $36(60.0)$ & $16(26.7)$ & $8(13.3)$ \\
API 20E profile & 0040524 & 0100004 & 0040004 \\
Result and likelihood & Pasteurella & Pasteurella spp. & Vibrio \\
level (\%) & multocida & (58.4) & hollisae \\
& $(99.9)$ & Pseudomonas & $(80.6)$ \\
& & cepacia $(12.0)$ & \\
& & Shewanella & \\
& & putrefaciens & \\
& & (10.2) & \\
& & Moraxella spp. & \\
& & Pasteurella & \\
& & multocida $(3.8)$ \\
\end{tabular}

excellent identification, with a $99.9 \%$ likelihood level and without tests against). Pattern II (0100004) was identified as either P. multocida or Pasteurella spp. (identities of $3.8 \%$ and $58.4 \%$, respectively); in addition, three other organisms were proposed (Pseudomonas cepacia, Shewanella putrefaciens and Moraxella spp., with identity rates of $12.0 \%, 10.2 \%$ and $9.6 \%$, respectively), and the API $20 \mathrm{E}$ database indicated that the profile identification was not valid for the 16 isolates comprised in this pattern. Finally, the remaining eight isolates (pattern III: 0040004) were misidentified as Vibrio hollisae, the profile identification being considered as acceptable. In our study, a phenotypic diversity concerning ornithine decarboxylase, indole, and fermentation of mannitol and sucrose has been obtained (Table 1), all being characters that have been considered as essential for the identification of $P$. multocida at the species level (Larivière et al., 1992). However, two recent studies have demonstrated that several isolates from diverse origins that had been regarded atypical for these four phenotypic characters, they were all ascribed genetically to $P$. multocida by $16 \mathrm{~S}$ rRNA gene sequencing and DNA-DNA hybridization (Christensen et al., 2004, 2005).

The biochemical reactivity of the $P$. multocida isolates examined here was quite different from the API 20E results previously reported for 14 paired isolates recovered from the lungs and kidneys of the same slaughtered pigs in Denmark and Canada, all giving an identical pattern (0144524; excellent identification, identity of 99.9\%) (Buttenschøn and Rosendal, 1990). However, our study was focussed on the evaluation of the identification capacity of the API 20E system at the species level, while that of Buttenschøn and Rosendal (1990) was undertaken to evaluate this system with sub-typing purposes. On the other hand, our results closely agreed with those obtained by Collins et al. (1981), with only $64 \%$ of 50 strains of $P$. multocida isolated from different animals being accurately identified by the API $20 \mathrm{E}$ system. In view of our results, the use of API 20E for identification of this organism at the species level is considered unsatisfactory, and the widespread use of this commercial system in the routine identification in clinical laboratories might therefore lead to misidentification of porcine $P$. multocida infection.

Concerning the API 20NE strips, 57 field isolates (95\%) and the reference strain did not assimilate glucose and mannitol, and their numerical code (3000004) allowed us to ascribe them to $P$. multocida, the identification level being good (accuracy of $93.1 \%$ ). In contrast, the remaining three isolates were positive to the two aforementioned tests (3044004), and the second pattern obtained in the API $20 \mathrm{NE}$ system yielded a low discrimination in the correct identification of this species, with an accuracy of only $42.9 \%$ for $P$. multocida. Besides, these isolates were also proposed to be representatives of Aeromonas salmonicida (identity of $55.2 \%$ ). However, these three isolates were correctly ascribed to $P$. multocida by the API $20 \mathrm{E}$ system (pattern I).

The API 20NE system has been developed for gramnegative organisms other than Enterobacteriaceae from 
human and veterinary sources and, for this reason, this is the method used routinely in veterinary diagnostic laboratories for the identification of $P$. multocida. The fact that all the 24 isolates unsuccessfully identified by the API $20 \mathrm{E}$ system were correctly ascribed to P. multocida by the API $20 \mathrm{NE}$ clearly confirms that this latter micromethod is more useful for an accurate identification of $P$. multocida isolates recovered from porcine lungs. In contrast, in a previous study carried out using a considerably lower amount of $P$. multocida strains (only six) isolated from rodent and rabbits, the species-level identification by the API 20NE system was considered unreliable by the four different diagnostic laboratories which profiled these isolates simultaneously (Boot et al., 2004).

By using the API $50 \mathrm{CHB} / \mathrm{E}$ system, all isolates fermented galactose, glucose, fructose, mannose, mannitol, sorbitol and $\mathrm{N}$-acetyl-glucosamine, while most of them fermented sucrose $(96.7 \%)$ and trehalose $(53.3 \%)$. However, most were negative by the ribose $(93.3 \%)$ and D-xylose $(83.3 \%)$ tests, and all isolates were nonreactive for the remaining carbohydrates included in this system. Variations in the patterns of fermentation of trehalose and D-xylose have been previously found for porcine P. multocida isolates (Unchitti et al., 1992; Blackall et al., 1997; Borowski et al., 2002). On the other hand, the different behaviour exhibited by mannitol and sorbitol in the API $20 \mathrm{E}$ compared to the API $50 \mathrm{CHB} / \mathrm{E}$ system could be related with the different preparation of inocula and incubation conditions.

On the basis of ribose, D-xylose, sucrose and trehalose results, the isolates were assigned to six profiles (Table 2), IV and $\mathrm{V}$ being the most frequent, with $40 \%$ of isolates each. The heterogeneity of carbohydrate profiles, along with the seventh one exhibited by the reference strain (which did not share phenotypic pattern with any of the clinical isolates), seem to suggest that this method, originally intended for the identification of other gram-negative organisms such as Enterobacteriaceae and Vibrionaceae, is unable to present a cohesive identification at the species level for P. multocida, the main species of Pasteurellaceae, a family also included into the class III of proteobacteria along with the two aforementioned families. However, such

Table 2

Biochemical patterns of 60 porcine Pasteurella multocida isolates yielded by the API $50 \mathrm{CHB} / \mathrm{E}$ system

\begin{tabular}{|c|c|c|c|c|c|}
\hline \multirow[t]{2}{*}{ Pattern } & \multicolumn{4}{|c|}{ Fermentation of: } & \multirow{2}{*}{$\begin{array}{l}\text { Number of } \\
\text { isolates }(\%)\end{array}$} \\
\hline & Ribose & D-xylose & Sucrose & Trehalose & \\
\hline I & + & + & + & + & $4(6.7)$ \\
\hline II & - & + & + & - & $4(6.7)$ \\
\hline III & - & + & + & + & $2(3.3)$ \\
\hline IV & - & - & + & + & $24(40.0)$ \\
\hline V & - & - & + & - & $24(40.0)$ \\
\hline VI & - & - & - & + & $2(3.3)$ \\
\hline $\begin{array}{l}\text { VII (reference } \\
\text { strain NCTC } \\
\text { 10322) }\end{array}$ & + & + & + & - & - \\
\hline
\end{tabular}

diversity in the API $50 \mathrm{CHB} / \mathrm{E}$ profiles could be a potential tool with epidemiological purposes, for characterising $P$. multocida isolates below the species level.

In the API ZYM, all isolates showed positive activities for alkaline and acid phosphatases, leucine arylamidase and naftol-AS-BI-phosphohydrolase, while $21.7 \%$ gave a positive $\alpha$-glucosidase reaction. On the other hand, none of the isolates produced esterase $(\mathrm{C} 4)$, esterase lipase (C8), lipase (C14), valine arylamidase, cystine arylamidase, trypsin, chymotripsin, $\alpha$-galactosidase, $\beta$-galactosidase, $\beta$ glucuronidase, $\beta$-glucosidase, $N$-acetyl- $\beta$-glucosaminidase, $\alpha$-mannosidase and $\alpha$-fucosidase.

Therefore, only two profiles differing in $\alpha$-glucosidase activity were generated: pattern I ( $\alpha$-glucosidase negative), shared by $47(78.3 \%)$ isolates, and pattern II ( $\alpha$-glucosidase positive), shared by the remaining ones and the reference strain. Quite similar results have previously been reported using 14 paired isolates coming from slaughtered pigs in Denmark and Canada (Buttenschøn and Rosendal, 1990). A higher diversity, mainly related to esterase (C4), esterase lipase (C8) and valine arylamidase, has been found among 91 P. multocida isolates recovered from the lungs of calves that died of bovine respiratory tract disease (Purdy et al., 1997). The homogeneity of enzymatic results revealed by this micromethod in our study seems to suggest its ability as an additional method to the API 20NE for a confirmatory identification of $P$. multocida of porcine origin. In addition, this method requires only a 4-h incubation, which is an advantage in terms of rapid response.

Taking together the four API systems, a total of 20 phenotypic profiles were obtained (Table 3 ), the most frequent being pattern I I V I (15 isolates), followed by patterns I I IV I (6 isolates), and I II IV I and I II IV II (5 isolates

Table 3

Global phenotypic patterns of 60 porcine Pasteurella multocida isolates yielded by the four API systems simultaneously

\begin{tabular}{|c|c|c|c|c|}
\hline \multicolumn{4}{|c|}{ Pattern obtained for: } & \multirow{2}{*}{$\begin{array}{l}\text { Number of } \\
\text { isolates }(\%)\end{array}$} \\
\hline API $20 \mathrm{NE}$ & API $20 \mathrm{E}$ & API50CHB/E & API-ZYM & \\
\hline \multirow[t]{18}{*}{ I } & \multirow[t]{7}{*}{ I } & I & I & $4(6.7)$ \\
\hline & & II & I & $2(3.3)$ \\
\hline & & IV & I & $6(10.0)$ \\
\hline & & IV & II & $3(5.0)$ \\
\hline & & V & I & $15(25.0)$ \\
\hline & & V & II & $2(3.3)$ \\
\hline & & VI & I & $1(1.7)$ \\
\hline & \multirow[t]{7}{*}{ II } & II & I & $1(1.7)$ \\
\hline & & III & I & $1(1.7)$ \\
\hline & & III & II & $1(1.7)$ \\
\hline & & IV & I & $5(8.3)$ \\
\hline & & IV & II & $5(8.3)$ \\
\hline & & V & I & $2(3.3)$ \\
\hline & & VI & II & $1(1.7)$ \\
\hline & \multirow[t]{4}{*}{ III } & II & I & $1(1.7)$ \\
\hline & & IV & I & $3(5.0)$ \\
\hline & & V & I & $3(5.0)$ \\
\hline & & $\mathrm{V}$ & II & $1(1.7)$ \\
\hline \multirow[t]{2}{*}{ II } & \multirow[t]{2}{*}{ I } & IV & I & $2(3.3)$ \\
\hline & & V & I & $1(1.7)$ \\
\hline
\end{tabular}


each). These results confirm the high diversity showed by $P$. multocida isolates, previously reported by others (Christensen et al., 2004, 2005).

In conclusion, the great phenotypic variability observed among the 60 porcine $P$. multocida isolates limits the usefulness of the API $20 \mathrm{E}$ and API $50 \mathrm{CHB} / \mathrm{E}$ systems for a correct identification at the species level. However, the API ZYM could help the API 20NE for a confirmatory identification of swine $P$. multocida infections in veterinary diagnostic laboratories, and the API $50 \mathrm{CHB} / \mathrm{E}$ system (or the four systems used simultaneously) could be useful for characterising isolates below the species level, especially taking into consideration that $P$. multocida is genetically rather homogeneous (Christensen et al., 2004).

This study has been founded by project LE22/04 of the Department of Education and Culture, "Junta de Castilla y León”, Spain.

\section{References}

Avril, J.L., Donnio, P.Y., Pouedras, P., 1990. Selective medium for Pasteurella multocida and its use to detect oropharyngeal carriage in pig breeders. Journal of Clinical Microbiology 28, 1438-1440.

Blackall, P.J., Pahoff, J.L., Bowles, R., 1997. Phenotypic characterisation of Pasteurella multocida isolates from Australian pigs. Veterinary Microbiology 57, 355-360.

Blackall, P.J., Fegan, N., Pahoff, J.L., Storie, G.J., McIntosh, G.B., Cameron, R.D., O’Boyle, D., Frost, A.J., Bará, M.R., Marr, G., Holder, J., 2000. The molecular epidemiology of four outbreaks of porcine pasteurellosis. Veterinary Microbiology 72, 111-120.

Boot, R., van den Brink, M., Handgraaf, P., Timmermans, R., 2004. The use of the API 20NE bacteria classification procedure to identify Pasteurellaceae strains in rodents and rabbits. Scandinavian Journal of Laboratory Animal Science 31, 177-183.

Borowski, S.M., Ikuta, N., Lunge, V., Fonseca, A., Marques, E., Cardoso, M., 2002. Caracterização antigênica e fenotípica de cepas de Pasteu- rella multocida isoladas de pulmões de suínos com pneumonia e/ou pleurite. Pesquisa Veterinaria Brasil 22, 97-103.

Buttenschøn, J., Rosendal, S., 1990. Phenotypical and genotypical characteristics of paired isolates of Pasteurella multocida from the lungs and kidneys of slaughtered pigs. Veterinary Microbiology 25, $67-75$.

Christensen, H., Angen, Ø., Olsen, J.E., Bisgaard, M., 2004. Revised description and classification of atypical isolates of Pasteurella multocida from bovine lungs based on genotypic characterization to include variants previously classified as biovar 2 of Pasteurella canis and Pasteurella avium. Microbiology 150, 1757-1767.

Christensen, H., Bisgaard, M., Angen, Ø., Frederiksen, W., Olsen, J.E., 2005. Characterization of sucrose-negative Pasteurella multocida variants, including isolates from large-cat bite wound. Journal of Clinical Microbiology 43, 259-270.

Collins, M.T., Weaver, N., Ellis, R.P., 1981. Identification of Pasteurella multocida and Pasteurella haemolytica by API 20E, Minitek, and Oxi/ Ferm systems. Journal of Clinical Microbiology 13, 433-437.

De Jong, M.F., 1992. (Progressive) Atrophic rhinitis. In: Leman, A.D., Straw, B.E., Mengeling, W.L., D'Alliare, S., Taylor, D.J. (Eds.), Diseases of Swine. Iowa State University Press, Ames, pp. 414-435.

Larivière, S., Leblanc, L., Mittal, K.R., Martineau, G.P., 1992. Characterization of Pasteurella multocida from nasal cavities of piglets from farms with or without atrophic rhinitis. Journal of Clinical Microbiology 30, 1398-1401.

Pijoan, C., 1992. Pneumonic pasteurellosis. In: Leman, A.D., Straw, B.E., Mengeling, W.L., D'Alliare, S., Taylor, D.J. (Eds.), Diseases of Swine. Iowa State University Press, Ames, pp. 552-559.

Purdy, C.W., Raleigh, R.H., Collins, J.K., Watts, J.L., Straus, D.C., 1997. Serotyping and enzyme characterization of Pasteurella haemolytica and Pasteurella multocida isolates recovered from pneumonic lungs of stressed feeder calves. Current Microbiology 34, 244-249.

Townsend, K.M., Boyce, J.D., Chung, J.Y., Frost, A.J., Adler, B., 2001. Genetic organization of Pasteurella multocida cap loci and development of a multiplex capsular PCR typing system. Journal of Clinical Microbiology 39, 924-929.

Unchitti, K., Wongsawang, S., Saitanu, K., Thoongsuwan, S., 1992. Characteristics of Pasteurella multocida isolated from humans, swine and poultry in Thailand. Southeast Asian Journal of Tropical Medicine and Public Health 23, 520-525. 